\title{
Heritage Language Maintenance Among Second-generation Chinese-American Children in a Small Chinese Community
}

\author{
Daina Zhu ${ }^{1,}$, Peggy Hopper ${ }^{1}$, Gulinaer Kulaixi ${ }^{2}$ \\ ${ }^{1}$ Department of Curriculum, Instruction, and Special Education, College of Education, Mississippi State University, Starkville, Mississippi, \\ USA \\ ${ }^{2}$ School of International Trade and Economics, Xinjiang University of Finance and Economics, Urumqi, China
}

Email address:

dz158@msstate.edu (Daina Zhu),pfh7@colled.msstate.edu (P. Hopper),441061078@qq.com (G. Kulaxi)

${ }^{*}$ Corresponding author

To cite this article:

Daina Zhu, Peggy Hopper, Gulinaer Kulaixi. Heritage Language Maintenance Among Second-generation Chinese-American Children in a Small Chinese Community. International Journal of Education, Culture and Society. Vol. 5, No. 5, 2020, pp. 100-114.

doi: $10.11648 /$ j.ijecs. 20200505.13

Received: October 3, 2020; Accepted: October 19, 2020; Published: October 26, 2020

\begin{abstract}
Based on the findings of previous research conducted in Chinese-American children's heritage language (HL) maintenance in large Chinese communities in urban settings, looking at positive parental attitudes, weekend Chinese schools, and interaction within local heritage language communities, the authors of this article investigated the contexts and outcomes of Chinese-language learning for second-generation Chinese-American children, in a small Southern United States college town. Research questions included: (1) What are the parents' and grandparents' attitudes about HL maintenance and efforts for passing on their HL to their children? (2) How consistent are parents' and grandparents' attitudes toward HL maintenance and their efforts of passing on HL to their children? What are reasons for the consistencies or inconsistencies? (3) Who has better learning outcomes, children who attending community Chinese-language school, or children taught by grandparents or parents? Results indicated that parents with higher education background and stay in the United State longer tend to have discrepancy between their attitudes and the real effort toward Chinese heritage language (CHL) maintenance. However, grandparents maintain consistency between their positive attitudes and the real effort regarding CHL maintenance. Grandparents' heritage language communication supported children's heritage language maintenance. Success of heritage language maintenance was determined more by children's communicative need than by parental attitudes or schooling.
\end{abstract}

Keywords: Chinese Heritage Language Maintenance, Second Generation Chinese-American Children, A Small Chinese Community

\section{Introduction}

Despite the best intentions to promote Chinese heritage language (CHL) maintenance, community Chinese-language schools (both Saturday schools and after-school programs) [1, 2], Chinese parents [1, 3-8] and grandparents [9] come across many difficulties and hardships on the way to develop Chinese immigrant children's competence of CHL even if in large Chinese communities. For example, both government-instituted and volunteer-instituted Chinese schools face similar challenges, such as funding issues, teacher recruiting and training, curriculum design, and many others [1]. In addition, previous research demonstrates that knowledge that Chinese immigrant children acquired from the Chinese-language schools did not get reinforced at home because of the lack of support from their parents [3]. Reference [3] further argues that neither Chinese-dominant parents nor English-dominant parents in immigrant families provide their children adequate exposure to both oral and written Chinese at home. For the Chinese immigrant parents' part, other study indicates that although Chinese immigrant parents maintained strong positive attitudes toward CHL learning, such as willing to contribute considerable time, money and energy to assist their children to learn Chinese, they still failed to pass down their positive family language attitudes they themselves keep to their children [8]. Researchers also find out that as primary caregivers in 
Chinese traditional culture, Chinese grandparents played a significant role in developing and maintaining their grandchildren's CHL [8, 9]. For example, Chinese grandparents started to develop their grandchildren's Chinese literacy at an early age with various ways, such as talking to them in Chinese, teaching them reading and writing in Chinese. However, [8] further suggests that Chinese immigrant children's CHL development gives way to the intergenerational conflicts, such as the disagreement on CHL development between Chinese immigrant parents and Chinses grandparents [9]. Additionally, some Chinese grandparents with limited educational background had limited practice of developing their grandchildren's CHL [9].

With $43 \%$ of Chinese immigrants residing in three metropolitan areas (New York City, San Francisco, and Los Angeles) from 2014 to 2018 in the United States [10], there is still a majority of Chinese immigrants live in non-metropolitan areas (Table 1). Unfortunately, CHL development in non-metropolitan areas remains under-researched. Additionally, research on CHL often fails to concurrently address all three aspects, community Chinese-language schools, Chinese immigrant parents, and Chinese grandparents, particularly relating to Chinese immigrant parents' and Chinese grandparents' attitudes toward CHL and the real effort they put in developing their children's CHL resonate with the instructional practice of community Chinese-language schools in the small Chinese communities. This is deeply troubling because (a) with limited Chinese language resources, the success of CHL maintenance in small Chinese communities (less than 2,500 residences defined by economic research service of United States Department of Agriculture) is even harder and relies more on family support and parents' attitudes and (b) without an advantageous heritage language environment in small Chinese communities [11], Chinese families who choose to provide instruction to their children at home by parents or grandparents, rather than enrolling in a Chinese school, take more responsibility for their children's HL maintenance.

Table 1. Top Concentrations by Metropolitan Area for Chinese Immigrants, 2014-18 [10].

\begin{tabular}{lll}
\hline Metropolitan Area & Immigrant Population from China & \% of Metro Area Population \\
\hline New York-Newark-Jersey City, NY-NJ-PA & 478,000 & 2.4 \\
San Francisco-Oakland-Hayward, CA & 264,000 & 5.6 \\
Los Angeles-Long Beach-Anaheim, CA & 262,000 & 2 \\
San Jose-Sunnyvale-Santa Clara, CA & 92,000 & 4.6 \\
Boston-Cambridge-Newton, MA-NH & 90,000 & 1.9 \\
Chicago-Naperville-Elgin, IL-IN-WI & 72,000 & 0.8 \\
Seattle-Tacoma-Bellevue, WA & 65,000 & 1.7 \\
Washington-Arlington-Alexandria, DC-VA-MD-WV & 59,000 & 1 \\
Houston-The Woodlands-Sugar Land, TX & 52,000 & 0.8 \\
Philadelphia-Camden-Wilmington, PA-NJ-DE-MD & 49,000 & 0.8 \\
\hline
\end{tabular}

What is more, different social and linguistic theories or systems were applied in the research on the dynamics between family support and CHL maintenance [12], such as ethnolinguistic identity [13], family system theory [14, 15], political perspectives [8], and Bourdieusian sociological perspectives [6, 16, 17]. It is worth mentioning that [9] integrated the theory of individual networks of linguistic contact with psychological and sociological frameworks and revealed the limited family support in CHL development in the United States. Taken together, there is an emerging body of perspectives regarding individual, familial and societal dynamics of CHL maintenance in English-speaking western countries. Yet, given the diversity of Chinese immigrant family backgrounds $[1,3,8,18]$, the reasons behind Chinese immigrant parents' and Chinese grandparents' attitudes toward CHL maintenance and the real effort they put in developing $\mathrm{CHL}$ need more investigation from a cultural-ecological perspective, i.e. Ogbu's cultural-ecological theory.

Consequently, there is an urgent need to develop a new avenue of investigation. One promising starting point is to investigate how community Chinese-language schools, Chinese immigrant parents, and Chinese grandparents contribute to Chinese immigrant children's CHL development in small Chinese communities. This type of research is especially crucial because (a) almost all of the Chinese immigrant children had some educational experience with community Chinese-language schools [19]; (b) research on CHL maintenance in metropolitan areas [11], such as New York City, indicates that the levels of heritage language (HL) development are different with regarding to the size of the HL communities; (c) although the diversity of Chinese immigrant families was frequently mentioned in previous studies $[1,3,8$, 18], limited research has been conducted with regards to a comparison of attitudes and real efforts toward CHL maintenance among parents with different professional backgrounds, and (d) in spite of the importance of the language attitudes in the field of language acquisition and language use, a sparsity of research is conducted on understanding the immigrants' attitudes toward their primary languages [8]. The research in this study investigates attitudes and efforts of Chinese immigrant parents and Chinese grandparents, and the role of community Chinese-language schools regarding heritage language maintenance of second-generation Chinese-American children. The aim of the study is to inform individuals who would like to maintain their heritage language which methods and people play the most significance in this process.

The research questions that drive the study include:

What are the parents' and grandparents' attitudes about HL maintenance and efforts for passing on their HL to their children? 
How consistent are parents' and grandparents' attitudes toward HL maintenance and their efforts of passing on HL to their children? What are reasons for the consistencies or inconsistencies?

Who has better learning outcomes, children who attending community Chinese-language school, or children taught by grandparents or parents?

\section{Literature Review}

\subsection{Ogbu's Cultural-Ecological Theory}

This current study investigates HL maintenance of second-generation Chinese-American children in a small Chinese community from perspectives of Chinese immigrant parents and Chinese grandparents under Chinese traditional culture and value systems. A crucial opening of this study is a discussion of Ogbu's cultural-ecological theory that undergirds the relationship between parents' attitudes and roles towards CHL and their immigrant background as well as their cultural background.

For those identified as first-generation immigrants with solid Chinese cultural backgrounds, [20] developed a cultural-ecological theory to explain the differences in immigrants' school performances. Reference [20] divided Chinese immigrants in the United States into three classifications, autonomous, voluntary immigrant, and involuntary immigrant. Chinese-Americans with academic working backgrounds were defined as voluntary immigrants because they voluntarily chose to move to the United States for the sake of better economic status and better education for subsequent generations. According to [21], culture and language differences could not completely explain their perspectives on the conflict between English learning and their group identity, but, the ways and reasons of their coming to the United States could. As voluntary immigrants, Chinese-Americans were more eager to accommodate and accept dominant culture and language in order to improve their economic status [20, 22]. Some researches [23, 24] advocated that besides the emphasis on efficacy of effort, Chinese-Americans were more likely to consider the discrimination as temporary and attributed it to their limited English proficiency. For this reason, they regarded learning English as adding a new language skill rather than eliminating their native language ability [21]. Furthermore, they did not see English learning at odds with their native language [25].

Indeed, Ogbu [21] further noted that Chinese-American parents considered basic English as a necessary requirement for their children's academic and occupational success. This consideration leads parents to assign a majority of their children's home-learning time to academic work rather than learning Chinese.

\subsection{Heritage Language}

No consensus exists of an accurate, scientific definition of a HL learner [26]. In the United States, HL refers to a language spoken by non-English speakers, working as a synonym for community or native language and mother tongue [27]. The HL learner described by [28] in pedagogical context matches the individuals investigated in this study. Reference [28] defines a HL learner as "someone who is raised in a home where a non-English language is spoken and who speaks or at least understands the language and is to some degree bilingual in the home language and in English" (p. 38).

Reference [29] took this definition a step further by ranging the HL learners from fluent native speakers to non-native-speakers. The latter are learners who may be removed from home countries for generations but still feel culturally tied to the language. Reference [29] notes the distinction between HL learners who have achieved a certain level of HL proficiency and/or have been raised in families with strong cultural connections with their home countries, and those HL learners who have heritage motivation and "seek to reconnect with their family's heritage language, even though the linguistic evidence of that connection may have been lost for generations" (p. 222).

Hence for the purposes of this current study, the authors followed the definition proposed by both [28, 29]. The individuals explored in this study are second-generation Chinese immigrants raised in families with two Chinese-native-speaker parents, maintaining strong cultural connections with China. They tend to be called CHL learners because their Chinese proficiency is below native level, but they are engaged in learning Chinese at a local community Chinese-language School.

\subsection{Family Support and Parents'Attitudes}

A myriad of research highlights the significant role of family support in promoting CHL development in western countries $[3-6,15]$. In the same vein, researchers explored the dynamics of family support of CHL development in the United States [30, 31] and described the bilingual competence (Chinese-English) among Chinese young children in the domestic sphere [4, 32-34]. Both quantitative [35] and qualitative studies [27, 36, 37] constructed a positive link between family support and CHL proficiency. Nevertheless, on one hand, [6] argues that more perceived and longer the family support promote young Chinese immigrant children more proficient in CHL. On the other hand, they believe that CHL development is the result of habitus. With Chinese immigrant children getting older, the resistance of learning CHL eliminates.

More specifically, previous studies emphasize the importance of family language policy in heritage language maintenance [38-40]. In addition, [3] considers language practice at home as the most crucial element in heritage language maintenance across generations. Consistently, [4] demonstrates a beneficial relationship between parents' positive attitudes and supportive domestic parent-child interactions, and Chinese immigrant children's primary language development. In the same vein, [8] describes great efforts that the Chinese immigrant parents put in their children's CHL development, such as practicing severe home language policy, becoming $\mathrm{CHL}$ teachers, participating 
cultural activities in the local Chinese community, and supporting their children to attend community Chinese-language schools. Considering participants' educational and professional backgrounds in [8], parents with higher educational background with more decent income tend to provide more positive support on their children's CHL development in the context of large Chinese community. According to [3], Chinese-dominant parents maintain higher expectations on their children's CHL level than that of the English-dominant parents. Reference [3] also reported an inconsistency between both Chinese-dominant and English-dominant parents' attitudes toward Chinese-language use at home and their real practice of home language use. Reference [3] attributes this attitude-effort inconsistency to the limited Chinese proficiency levels of the parents. However, [4] argues that regardless of parents' proficiency in English and Chinese, their positive attitudes toward CHL maintenance play an important role on their children's perspectives on CHL learning.

Ogbu's Cultural-Ecological Theory advocates that parents' working backgrounds from the immigrant families decide the significance of family dynamics and socialization patterns for immigrant children's native language development [16, 17]. Regarding these children's HL maintenance, family support and parents' attitudes toward their language of origin have had a significant effect on their HL proficiency levels [41]. Most of the research done in this area has emphasized the positive correlation between positive attitudes toward one's mother tongue and the speaker's proficiency level [38, 42]. As for family support, a family HL policy and formal home-based instruction are considered two essential factors [6]. Moreover, since grandparental involvement in early childcare attributes Chinese traditional culture [43], grandparental contribution to children's CHL maintenance should not be ignored.

\subsection{Grandparents'Attitudes Toward CHL Development}

Although previous studies mentioned intergenerational interaction as a factor in CHL maintenance, for example, one of the participants in [6] considered the encouragement provided by her uncle as a positive element for her CHL learning, as primary caregiver in Chinese families, grandparents' perspectives related to $\mathrm{CHL}$ development received limited investigation. One exception is [9], which focuses on analyzing grandparents' perspectives on CHL maintenance, and makes comparison with Chinese immigrant parents' attitudes toward CHL development in a large Chinese community of a metropolitan area in Canada. Reference [9] reported that the Chinese grandparents took the responsibility of both transmitting Chinese language and conveying Chinese traditional culture and values. Reference [9] further noted that Chinese immigrant families with grandparents were more likely had more exposure to Chinese, such as the subscription of Chinese TV channels, story books in Chinese, and other learning materials. Compared to the Chinese immigrant parents who can speak both English and Chinese, bilingual grandparents felt more comfortable in communicating with their grandchildren in Chinese [8, 9]. As suggested by [9],
English proficiency did not prevent Chinese grandparents from practicing CHL maintenance, it actually facilitated the development of their grandchildren's CHL. Chinese grandparents played a significant role on developing their grandchildren's Chinese literacy skills, especially oral proficiency [9].

Despite positive attitudes toward CHL maintenance, Chinese grandparents had some concerns regarding CHL learning [9]. For example, Chinese immigrant children might come across confusion by learning Chinese pinyin (Chinese phonetic symbols written in Roman letters) and English alphabets. Other studies document the same concerns held by Chinese immigrant parents [44-46]. In addition, some grandparents had the concerns that learning Chinese would reduce their grandchildren's interest in learning English. Contrary to the Chinese immigrant parents with higher education background and satisfied-income, Chinese grandparents did not impose strict family language policy. Instead, they were proud of their bilingual grandchildren [9]. Based on the interview data of [9], even though providing the primary caregiving in Chinese families and maintaining very intimate relationship with their grandchildren, Chinese grandparents still were not the decision maker of the family. On the other hand, the Chinese immigrant parents, as the main contact to the local Chinese community, involved more in children's academic study and making decisions of the children's future [9].

\subsection{Community Chinese-Language Schools}

Another indispensable source of CHL is the 130-year-old history of CHL schools [2, 47]. Besides these official organizations, there are non-profit CHL schools in small Chinese communities which are operated primarily by volunteers including parents of enrolled students and students from local high schools, colleges, and universities [1, 18, 48-50]. Funding for these non-profit schools is mainly collected from tuition and fundraising [1].

CHL schools in United States normally operated by local communities become widespread and vigorous recent years and play a decisive role in CHL maintenance. CHL schools depend largely on community parents even in large Chinese communities [1]. Particularly, community parents who are interested in their primary language development often send their children to CHL schools and serve active roles on school administration $[1,50]$. It is an unspoken expectation that parents are responsible for the success of the CHL schools and are expected to assist schools in different ways, such classroom teaching or helping with learning activities [1, 51]. Although [9] suggests that Chinese immigrant parents' active role in CHL schools facilitates their children's HL learning, previous studies consistently argue some drawbacks caused by the over involvement of the Chinese parents in CHL schools, such as lack of generally agreed teacher recruitment criteria, high turnover rate and unqualified teachers [1, 48-50, 52]. Besides, CHL schools also suffer from limit selection of textbooks (e.g., [53]). 


\section{The Current Study}

Taken together, the first line of literature on family support and parents' attitudes in the context of large communities toward CHL maintenance suggests that (a) there is a positive link between family support and CHL proficiency (e.g., [6]), (b) parents' educational and economic background tend to impact on their support on their children's HL development (e.g., [8]), and (c) there is an inconsistency between parents' attitudes and real effort they put on CHL maintenance based on their primary language proficiency (e.g., [3]). Comparing with Chinese immigrant parents' attitudes and efforts toward CHL, the second line of literature on grandparents' perspectives on CHL development suggests that Chinese grandparents played a significant role on developing their grandchildren's Chinese literacy skills, though concerns do exist (e.g., [9]). However, although the second line of literature made comparison between parents and grandparents, it fails to investigate and compare the effectiveness of parents' and grandparents' domestic inculcation of CHL, whereas the first line of literature tends to overlook how educational and economic background influence immigrant parents' support to their children's CHL learning and fails to investigate the relationship between parents' educational and economic background, and the inconsistency between their attitudes and real effort they have towards CHL. Finally, the third line of literature on community Chinese-language schools implies that CHL preservation largely depend on Chinese communities, especially Chinese schools (e.g., [1]). Parents' active involvement decides the success of the school, meanwhile, their over investment raises issues, such as high turnover rate of teachers and unqualified teachers (e.g., [52]). However, the effectiveness of CHL schools, especially in small Chinese communities, has not been investigated.

In addition, all the three lines of literature have primarily studied large Chinese language communities in urban settings, leaving open the question of influences on language maintenance in smaller communities. Furthermore, although qualitative research has been conducted to explore parents', grandparents', and children's attitudes toward HL learning, no quantitative research has been done to compare the teaching outcomes of parents, grandparents, and Chinese schools. Rare exceptions to the lack of quantitative evidence include studies $[6,11,54,55]$, but evidence only included individual self-reports of Chinese as a second language proficiency level instead of standardized Chinese proficiency test scores. Consequently, this study fills current research gaps by setting the study in a small Chinese community and addressing the dearth of quantitative evidence on CHL teaching outcome comparisons of parents, grandparents, and CHL schools. In addition, this study investigates the reasons for perceived gaps between parents' attitudes and their real effort on children's HL maintenance.

\section{Methods}

This study was conducted in a small Chinese community, located in a small college town in Mississippi with a population of 25,543 [56]. This college town is an appropriate setting for this study since Asian people in this small town consist of $3.93 \%$ of the population [56] and Asian students only consists of $1.09 \%$ of the local school district student population [57]. There is no available official Chinese demographic information of this college town, but obviously, it is a small Chinese community based on the size of Asian population. There is only one nonprofit weekend Chinese school in this college town to promote Chinese language and culture among the local Chinese communities. This school was informal as it was founded voluntarily by Chinese parents in 2003. Parents with children enrolled at this school take turns to serve as administrative staff and teachers every year. It normally provides one two-hour class per week, 12 times each semester. There were 30 students in total enrolled at this school attending five classes based on different Chinese proficiency levels at the time when this research was conducted.

A mixed-method study was conducted in order to provide a comprehensive understanding of the research questions [58]. Perspectives from parents, grandparents, and Chinese teachers on CHL maintenance in a small Chinese community constructed the data triangulation of this study. The researchers analyzed data from surveys and interviews with parents/grandparents and Chinese teachers, and observations of Chinese classes. Due to the small sample size participants of this study, scores of a standardized Chinese proficiency test was utilized as supplement data for the purpose of filling the gaps of lacking quantitative data in previous research.

A survey was given to the cohort of parent, grandparent, and teacher participants at the onset of the study. One 60-minute, semi-structured interview was conducted based on the results of the survey with 10 chosen adult participants for the purposes of including diverse backgrounds of participants and exploring a deep understanding of their attitudes toward CHL maintenance and the real efforts they put on their children's/grandchildren's Chinese learning. Interviews were conducted in Mandarin or English based on the participants' preference. The researcher translated and transcribed the interviews verbatim then shared the translation and transcripts with the participants for checking translation accuracy [59]. Four one-hour Chinese class observations were conducted at two different classes along one semester, with detailed observational and reflective field notes [60].

\subsection{Participants}

Given the validity of the standardized Chinese proficiency test results and difficulties of using probability sampling strategies in the small Chinese community, the researcher adopted snowball sampling, which is a widely used strategy in HL research [54, 61, 62].

There are 30 second-generation Chinese-American participants ( 18 boys, 12 girls) ranging in age from 6 to 8 years $(M=7.10 ; S D=.76)$. Due to small Chinese population of this college town (according to the data provided by the administrative staff of the Chinese school, there are only 34 second-generation Chinese-American children aged from 6 to 
8 in the whole town), these 30 children participants cover a majority of this age group and could represent a general situation of CHL maintenance of this age range. The number and gender of children of each age is similar in each group (see Table 2). Among all participants, ten attended Chinese weekend school regularly, ten children did not attend any Chinese school but were taught by their grandparents, and ten were taught by their parents at home without attending Chinese weekend school. For the 20 children who did not attend the Chinese school when the research was conducted had taken some Chinese school classes for different length of time ranged from one day to three months. However, they stopped to go to Chinese school for different reasons. In addition, the groups' divisions have overlapping situations. Children of the school-taught group might be instructed by their parents or grandparents at home as well. At times, the children of the parent-taught group might sometimes visit their grandparents who may provide Chinese instruction during their visitation. For the grandparent-taught group, children may have had Chinese learning instruction from their parents. Therefore, in this study, children's groups were divided depending on their primary Chinese learning situation. To answer the third research question, children participants in this study were invited to take a standardized Chinese proficiency test of a certain level. It is hoped that a narrow age range of participants will eliminate threats to internal validity.

Table 2. Number and gender of children participants of each group.

\begin{tabular}{lllllll}
\hline & Age 6 & Age 7 & Age 8 & Male & Female & Total \\
\hline Group 1 & 4 & 4 & 2 & 7 & 3 & 10 \\
Group 2 & 2 & 4 & 4 & 5 & 5 & 10 \\
Group 3 & 4 & 3 & 3 & 4 & 6 & 10 \\
\hline
\end{tabular}

Group1=school-taught. Group2=grandparent-taught. Group3=parent-taught.

Six Chinese parents and four Chinese grandparents from ten families were interviewed (see Table 3 for family profile). In addition, two parent participants who also worked as Chinese teachers (see Table 4 for teacher profile) were interviewed as both parents and teachers in order to explore teaching outcomes of the Chinese Weekend School. For all the ten families, parents of eight families were affiliated to a university. The other two families all had business working background, one managing an Asian supermarket and the other running a Chinese restaurant in the college town. They immigrated to the United States with other family relatives who were doing resale or catering business in other cities of the United States. All the participants of this study were assigned pseudonyms.

Table 3. Chinese family profile.

\begin{tabular}{lllll}
\hline & Mother & Father & $\begin{array}{l}\text { Grandparents Provide } \\
\text { Chinese Instruction at home }\end{array}$ & Home Language \\
\hline Family 1 & Professor* & IT Staff & No & English dominant \\
Family 2 & Lecturer* & Professor & No & Yes \\
Family 3 & Staff & Professor & Yes & English dominant \\
Family 4 & Self-employed & Professor & Yes & Chinese dominant \\
Family 5 & Full-time Mom* & Professor & No & Chinese \& Shanghai Dialect \\
Family 6 & Researcher & Professor & Yes & Nominant \\
Family 7 & Graduate Student & Graduate Student & Yes & English dominant \\
Family 8 & Graduate Student* & Graduate Student & No & Chinese dominant \\
Family 9 & Businesswoman* & Businessman & No & Chinese dominant \\
Family 10 & Businesswoman* & Businessman & No & English dominant \\
\hline
\end{tabular}

+ Parents participating the interviews are marked with *. Parents who worked as a part-time Chinese teacher and participated the interviews as a Chinese teacher are marked with **. All the four grandparents who provided Chinese instruction at home participated the interviews.

Table 4. Chinese teacher profile.

\begin{tabular}{|c|c|c|c|c|c|c|}
\hline & Gender & Occupation & Educational Level & Major Field & $\begin{array}{l}\text { Teaching Experience } \\
\text { at Chinese School }\end{array}$ & $\begin{array}{l}\text { Chinese Enrolled } \\
\text { at Chinese School }\end{array}$ \\
\hline Chinese Teacher 1 & Female & Full-time mom & Master & Accounting & 1 Year & Yes \\
\hline Chinese Teacher2 & Female & Graduate Student & Master & Economics & 2 Months & Yes \\
\hline
\end{tabular}

\subsection{Procedure}

\subsubsection{Survey}

All parent participants were asked to complete a survey revised from a sample survey designed by [63]. Descriptive, behavioral, and preferential information were collected through the survey, including demographic and family background information, and how parent participants valued and treated their children's language learning. The results of the survey provided a general picture of participants' attitudes and efforts. Interview questions were then created based on survey results.

\subsubsection{Interview}

After viewing survey results, six parents and four grandparents were purposefully chosen to participate in a semi-structured interview based on their different working backgrounds. The settings of the interviews were either in 
participants' homes or their workplaces. Thus, the researcher had chances to observe home language usage in different social settings.

These two groups represented two main Chinese immigrant family types in the small Chinese community: Chinese traditional family of two Chinese parents with higher education backgrounds, and Chinese traditional family of two Chinese parents with business working backgrounds. Since two of the Chinese parents also worked at the Chinese Weekend School as teachers, they also participated in interviews as teachers following the four one-hour class observations to illustrate their teaching experiences and teaching outcomes.

\subsubsection{Test}

Youth Chinese Test (YCT) is an international standardized Chinese test organized by Hanban, which is a Chinese government organization focusing on the management of Confucius Colleges out of China and the promotion of Mandarin study. The 30 children participants completed YCT Band 2, which includes listening, reading, and speaking; each part totals 100 points, for a maximum score of 300 .

\subsubsection{Class Observation}

Four one-hour class observations were conducted in two different classes but with same Chinese proficiency level at the local Chinese Weekend School along one semester. Since there were only 30 students in total, in order to provide more communicative instructions, ten student participants with similar Chinese proficiency level was assigned to two classes. The teachers are parents of second-generation Chinese-American children and are fluent Chinese speakers without previous teaching experience.

The researchers observed two teachers' four one-hour classes at different times of the semester. Since there were 12 times of classes each semester, four times observation could provide a general picture of their teaching methodology and students' learning situations at the Chinese Weekend School. Observation tasks were focused on classroom management, teacher-student interactions, and purpose and design of classroom activities. The observation sheet used was a revision of a template established by [64].

\subsection{Data Analysis}

\subsubsection{Interview Analysis}

NVivo 11 was used to create and maintain an audit trail, a detailed record of when and how the interviews were conducted (e.g. coded interview transcripts), together with applicable comments made during each step of data analysis (e.g., memos). This detailed filing system enabled the process of data analysis adequately transparent, so that increasing the warranty of the findings.

Additionally, based on the results of the survey, the researchers investigated parents' and grandparents' attitudes toward HL maintenance and any real efforts they make on maintaining their children's HL. Furthermore, parent participants' occupations and residential information were included in the classification. With the help of word frequency, the researchers were able to identify what factors related to CHL maintenance the parents and grandparents mentioned more frequently. The researchers also employed four matrix requests. First matrix requests were made between parents' attitudes and efforts and grandparents' attitudes and efforts in order to draw more information on the relationship between attitudes and efforts. The other two matrix requests were made between classification information and parents' attitudes and real effort respectively, in order to see if parents' working background and the amount of time they lived in the United States made them think and act differently in CHL maintenance.

The data of the YCT test scores is used as a supplement data to indicate the learning outcome of each group of the students.

\subsubsection{SPSS Report of Children's YCT Test Data}

The sample for this study consists of 10 grandparent-taught children, 10 parent-taught children and 10 school-taught children $(n=30)$. To compare the performance of the three different groups of students on the following measures: Listening, Reading, Speaking, and the Total score of the three skills, a one-way multivariate analysis of variance (MANOVA) followed by separate univariate ANOVA, and a one-way repeated measures ANOVA were employed.

Table 5 illustrates the mean and standard deviation of the three groups. It evidences that the grandparent-taught group obtained higher means in Listening, Reading, Speaking, and Total with scores 94.5, 66, 96, 256.5 respectively when compared to the other two groups. Using Wilks's lambda, there is a significant effect of different learning environments on Chinese general skills of second-generation Chinese-American children, $\Lambda=0$. 501, $F(6,50)=3.441, p=.006$. The MANOVA was followed up with separate univariate ANOVAs on the outcome variables, indicating significant effects of different learning environments on the Listening test $F(2,27)=9.642, p=.001$, Reading test, $F(2,27)=6.267, p=.006$, Speaking test, $F(2$, $27)=5.889, p=.008$, and Total score, $F(2,27)=11.265, p$ $<.001$ (see Table 6). Based on the MANOVA results, it is concluded that the grandparent-taught participants in this study have higher scores on the Listening, Reading, Speaking, and Total parts than school-taught and parent-taught participants.

Mauchly's test indicated that the assumption of sphericity had been violated, $\chi^{2}(2)=10.215, p=.006$, therefore Huynh-Feldt corrected tests are reported $(\varepsilon=.800)$. Results of one-way repeated measures ANOVA show that there is a significant score difference among the three skill of CHL, $F$ $(1.6,46.404)=237.726, p<.001, \eta_{\mathrm{p}}^{2}=.891$. Post hoc test (Bonferroni) reveals that there is not significant difference between listening skill $(M=86.83, S D=8.785)$ and speaking skill $(M=89.87, S D=8.401 ; p=.05)$, but reading skill $(M=56.83, S D=11.853)$ is significantly lower than either listening skill $(p<.001)$ or speaking skill $(p<.001)$. 
Table 5. Descriptive statistics.

\begin{tabular}{|c|c|c|c|c|c|c|c|c|}
\hline & \multicolumn{2}{|c|}{ Listening } & \multicolumn{2}{|c|}{ Reading } & \multicolumn{2}{|c|}{ Speaking } & \multicolumn{2}{|l|}{ Total } \\
\hline & $M$ & $S D$ & $M$ & $S D$ & $M$ & $S D$ & $M$ & $S D$ \\
\hline Group1 & 84.50 & 8.96 & 51.00 & 11.01 & 87.50 & 7.91 & 223.00 & 21.88 \\
\hline Group2 & 94.50 & 4.97 & 66.00 & 9.66 & 96.00 & 6.58 & 256.50 & 17.33 \\
\hline Group3 & 81.50 & 6.26 & 53.50 & 9.73 & 85.50 & 7.25 & 220.50 & 17.23 \\
\hline Total & 86.83 & 8.76 & 56.83 & 11.85 & 89.67 & 8.40 & 233.33 & 24.75 \\
\hline
\end{tabular}

Table 6. MANOVA and ANOVAs results.

\begin{tabular}{|c|c|c|c|c|c|}
\hline Effect & Wilks' & F-value & $d f$ & Error $d f$ & $p$ \\
\hline $\begin{array}{l}\text { Main effect } \\
\text { Separate effect }\end{array}$ & .501 & 3.441 & 6 & 50 & .006 \\
\hline Listening & & 9.642 & 2 & 27 & .001 \\
\hline Reading & & 6.267 & 2 & 27 & .006 \\
\hline Speaking & & 5.889 & 2 & 27 & .008 \\
\hline Total & & 11.265 & 2 & 27 & $<.001$ \\
\hline
\end{tabular}

\section{Findings and Discussions}

\subsection{Attitudes and Real Efforts Toward HLM}

\subsubsection{Parents vs. Grandparents}

First, the results of the survey revealed an obvious discrepancy between parents' attitudes toward HL maintenance and their real effort to support their children's HL learning; however, grandparents' attitudes toward HL maintenance was consistent with their real efforts in passing the HL to children. For example (see Table 7), there are several questions asking how often the parents read or watch TV with their children in Chinese or English. The answers of these questions are surprisingly similar among parents. For Chinese, they all chose less than once a week, but for English, all chose two or more than three times per week. These answers align with what the researcher's findings through interviews, which is parents' limited effort of passing on HL to their children and their emphasis on English study rather than Chinese study. However, the grandparents weighed equally between Chinese and English study.

Second, unlike the parents, grandparents' positive attitudes toward HL learning were reflected in their active role and effort in instructing Chinese to their grandchildren. For example, for "Please choose all the things you do to support your grandchildren's HL learning," grandparents chose: speaking Chinese at home, reading Chinese books, Chinese calligraphy, eating Chinese cuisine, and travelling to China, while only four parents chose speaking Chinese at home, eating Chinese cuisine, and eight parents chose speaking Chinese at home, eating Chinese cuisine and travelling to China.

Table 7. Result of survey questions related to home-learning time.

\begin{tabular}{|c|c|c|c|c|c|c|c|c|}
\hline Survey Questions & & & & & $3+$ & & $<1$ & \\
\hline Parents (P)/Grandparents (G) & $\mathbf{P}$ & $\mathbf{G}$ & $\mathbf{P}$ & G & $\mathbf{P}$ & $\mathbf{G}$ & $\mathbf{P}$ & $\mathbf{G}$ \\
\hline I read Chinese with my child__ per week. & 0 & 2 & 0 & 2 & 0 & 8 & 14 & 0 \\
\hline I watch Chinese movie/TV with my child_ per week. & 0 & 4 & 0 & 8 & 0 & 0 & 14 & 0 \\
\hline I listen to Chinese radio broadcast with my child_ per week. & 0 & 6 & 0 & 6 & 0 & 0 & 14 & 0 \\
\hline I read English with my child__ per week. & 0 & 2 & 4 & 2 & 10 & 8 & 0 & 0 \\
\hline I watch English movie/TV with my child_ per week. & 0 & 4 & 4 & 8 & 10 & 0 & 0 & 0 \\
\hline I listen to English radio broadcast with my child & 0 & 6 & 0 & 6 & 14 & 0 & 0 & 0 \\
\hline
\end{tabular}

Third, Chinese grandparents with different educational background all keep positive attitudes and practice real effort toward CHL maintenance; whereas, as suggested by the NVivo matrix request, Chinese immigrant parents with higher educational background remain inconsistent between their attitudes and efforts toward CHL maintenance. Although Chinese immigrant parents expressed positively in CHL maintenance, the higher their educational backgrounds are, the less likely they will put real effort on their children's Chinese learning, such as establishing home language policy, sending children to the Chinese weekend school. However, without explicit perspectives on HL maintenance, parents with business professional backgrounds only speak Mandarin or certain Chinese dialect at home because of their lack of
English fluency. Evidences were indicated by interview data.

\subsubsection{Grandparents}

Min, receiving her bachelor's degree in 1970s, is the grandmother of Family 6 (see Table 3 ). She came to visit her grandchildren once a year and stayed with them for at least five months each time. She got very strong and positive attitudes toward CHL maintenance, which is also consistent with her effort.

Excerpt 1

Min: No matter where my grandchildren were born, what nationality they are. Their race is categorized as Chinese. This will never change. Their parents, grandparents, all the family relatives, are all Chinese. This will never change. And they can't cut off the relationship. Therefore, instead of an interest. 
Learning Chinese is a necessity and a responsibility for them. They need to communicate with family members other than their parents. When they visit China each year, you know, they at least need some basic knowledge of Chinese. Additionally, China's world economic status is improving. Knowing Chinese might provide them more chances in the job markets in the future. It is hard to say. Anyway, it doesn't hurt to know a language other than English. In my opinion, raised up in a Chinese family without knowing Chinese is ashamed.

Min: Yes. I send my grandson to the Chinese school. My granddaughter is only 2 . She will go to the Chinese school too when she is old enough. We live in a very small Chinese community. Chinese people are very close and know each other. It is good. Going to Chinese school will give him a very good context of learning Chinese. Although Lele (the name of her grandson) doesn't like it, but he will know how and what he benefits from attending Chinese school one day. Learning a language is a life journey. I am sure he won't regret in the future. I teach him at home. I always bring a lot of Chinese learning materials and Chinese story books from China. My daughter and son-in-law are busy at work all day. So I need to take more responsibility for my grandchildren's CHL maintenance.

Ying, another grandmother participant from Family 7 (see Table 3), often visit her grandchildren in this small college town. Although Ying's son and daughter-in-law were both $\mathrm{PhD}$ candidates of the local university, Ying herself only finished elementary school in China because of the ten-year Cultural Revolution. Similar as Min, Ying held very positive attitudes and put real efforts toward CHL maintenance.

Excerpt 2

Ying: I try my best to teach my granddaughter Chinese. I think this is the only thing I can teach to her. And it is very important to know Chinese, otherwise, we could hardly communicate with each other. I am 64. I never learned English at school. It is impossible for me to learn English at this age now. So the only way is let her learn Chinese. I am not doing the formal teaching. Learning happens in every communication between her and I.

The other two grandparent participants, both received their education in China, earned a university diploma and a high school diploma, respectively. However, no matter what educational background they have, they all keep a positive attitude toward CHL maintenance. Neither of the four grandparents had a specific curriculum or teaching plans for instructing their grandchildren Chinese. They all chose to naturally immerse the Chinese instruction to their daily life.

\subsubsection{Parents}

In an interview with the mother of Family 1 (see Table 3), both Jing and her husband are employed at a local university. They lived in the United States for almost 15 years and have an 8 -year-old daughter.

Excerpt 3

Researcher: What you think the importance for your daughter to learn Chinese?

Jing: Of course. It is important. You know, nationality and your original race are two different concepts. Though she is considered as an American since she was born here. However, she cannot deny that she has a strong relationship with China. We look like Chinese and all of my relatives are in China. If she doesn't know any Chinese, she will not be able to communicate with other family relatives when we are back to China. However, when thinking about Susan's (Jing's daughter) future, English and math are more important subjects for her, especially English. You know, since all the subjects are taught in English. All the textbooks are written in English. I know how it suffers if English proficiency is limited, just like 15 years ago, when I first came to study in a university in northern United States.

Researcher: OK. So how do you usually promote her Chinese learning?

Jing: She goes to (Chinese) Weekend School.

Researcher: How about at home? Do you have any language policies at home?

Jing: Well. We don't.

Researcher: So you mean she speaks whatever language she prefers?

Jing: (Long hesitate) Yes.

Researcher: In that case, what language does she use more, Mandarin or English?

Jing: Half and half, I should say? (hesitate)

Researcher: What is your language preference? What language do you use when you start the conversation?

Jing: Most of the time, I use Mandarin. Sometimes, English.

Researcher: But you don't care what language your daughter uses when responding to you?

Jing: (Laughing)

Researcher: Do you?

Jing: (Laughing) No.

According to Jing's statement, there was a great difference between her attitudes of HL maintenance and her effort. In Excerpt 3, she expressed her positive attitudes explicitly. However, when asked about her real effort on promoting her daughter's Chinese learning, she hesitated several times, such as in Excerpt 3. Additionally, there was obvious embarrassment on her face when she was hesitating. Jing's own bittering English learning experience makes her consider English proficiency is utmost important. As non-English-native-speaker, she believes that English proficiency is warrant for a bright future.

In Excerpt 4, Lian and her husband (Family 9) (see Table 3) came to the United States 25 years ago and managed an Asian supermarket in the town. She speaks Hokkien, a Chinese dialect originating from the southeastern China, with her husband and a mix of Hokkien and Mandarin with her two elder children, but Mandarin-only to her younger son. When I arrived at her supermarket for the interview, she was talking to her younger son on the phone.

Excerpt 4

Lian: I couldn't go back home now.

Lian: There are customers in the store now.

Lian: Can your brother send you here?

Lian: He is still sleeping? My goodness. OK. Wait for me. 
Lian: Maybe 30 or 40 minutes?

Lian: Great. Bye-bye.

Researcher: So you speak Mandarin to your children?

Lian: Yes. I speak Hokkien to my husband, but I speak

Mandarin to my children.

Researcher: Hokkien? Oh, to me it is not a Chinese dialect, but totally a foreign language. (Laughing) Do your children speak Hokkien?

Lian: No. They could only understand some simple words. Just a little.

Researcher: So when you talk to your children, in what language will they respond?

Lian: Most of the time, they speak Mandarin. Unless they are $100 \%$ sure that I will understand their English response, they will speak English. Er... some very simple words.

Researcher: Are there any language shifts?

Lian: Sometimes. Not very often.

Researcher: Interesting. In what situations will they use English to communicate with you?

Lian: When they play computer games. For example, yesterday, Jason (a pseudonym), my younger child, was playing computer games. Suddenly, he shouted, "Mom, look! It is interesting!"

Researcher: Do your children go to Chinese Weekend School?

Lian: No. My husband and I are too busy. We have no time to send and pick them up. You know, Sundays are one of my busiest days. There are usually a lot of customers. In addition, I have no time to supervise their Chinese school assignment.

Researcher: Do you think it is important for your children to learn Chinese?

Lian: They know Chinese. They can listen and speak. They can communicate with the Chinese customers very often. When I was out, they can manage the business. They are fluent in Mandarin and English. (Laughing)

Researcher: Can they read and write Chinese?

Lian: No. But they don't need to read and write in Chinese very often, do they?

Contrary to Jing, Lian's attitude toward children's Chinese learning is optional. However, main languages used in her family are limited to Mandarin and Hokkien, which partially promote her children's HL maintenance. In Excerpt 4, Lian also laughed but with a different reason as Jing. Lian's laugh indicated her satisfaction with her sons' Chinese fluency. In addition, there was an immensely proud tone in this line.

Other parents who have similar familial backgrounds as Jing or Lian, also maintained similar responses in the interviews. In Family 2 (see Table 3), Rui is a laboratory assistant and her husband is a professor at the local university. They studied and worked in the United States for 17 years.

Excerpt 5:

Rui: I know learning Chinese is important. Though my daughter was born in the United States, she couldn't cut off her relationship with her relatives in China. But, you know, it is difficult. Anne is at the day care now, but her two elder brothers attended school for several years. They communicate in English at home. I couldn't stop them. So now, all three speak English. Though I speak Chinese to them, they reply in English. I know I should stop them from doing that, but I am tired. I am so tired after 8-hour working. I just let them go. They use English everywhere here in the United States, especially in schoolwork. So it is easy to tell. If Chinese and English are both important, English is more important. My husband and I spent more than ten hours to study English every day when we first came to the United States. We missed a lot of good chances because of our less proficient English, such as grant application, job promotions, and so on. I hope my children won't experience these.

In Family 10 (see Table 3), Hai and his wife, living in the United States for about 8 years, are the owners of a Japanese restaurant in the town. Their two children, a 7-year-old, and a 9-year-old, have been attending the Chinese Weekend School for two years. Hai's children are the only ones with business family background in the Chinese Weekend School.

Excerpt 6:

Hai: Yes. My friends help us send them to the Chinese

Weekend School every week.

Researcher: OK. So what else do you do to facilitate your children's Chinese study?

Hai: We speak Mandarin at home, most of the time.

Researcher: Most of the time? So you speak English at home as well?

Hai: Not that much. We might use English words in a sentence. For example, pass me the calculator. We speak Hokkien more than English at home actually. My kids understand some basic conversation in Hokkien. They don't speak any Hokkien, though.

Researcher: Do your children learn a lot from the Chinese Weekend school?

Hai: Look. I don't expect them to learn a lot there. My friend sends them there just because they will have some place to stay for two hours on Sundays, not bothering us at the restaurant. Sundays are one of our busy days of the week. You know, people usually eat out after the church.

With a business family background, the positive attitudes towards HL maintenance of Hai's family does not relate to their emphasis on Chinese language or culture maintenance. It has much to do with their language convenience and their working needs.

Another finding indicates that in light of the previous interview excerpts, parents' involvement in children's HL learning has loose relationships with their attitudes, but some relevance to their family background. Business working Chinese immigrant parents have more active and greater involvement, such as their daily communication at home and their children's exposure to Chinese, than university-affiliated Chinese immigrant parents. However, Hai's statements unintentionally conform to what [65] demonstrated in the research that parents' language choice would significantly influence their bilingual children's language preference. In addition, [5] demonstrates that parents' inconsistency is one of the main reasons of HL maintenance failure.

Previous findings are also supported by the NVivo matrix request. Among parents with university-affiliation, the longer 
the parents stayed in the United States, the less likely they were to contribute to family language policies, and the less positive attitudes they maintained for CHL learning. Moreover, the number of years that each Chinese family stayed in the United States was classified as1-5, 6-10, 11-15, and 16-20. In terms of the matrix request, the longer the family stayed in the United States, the less they emphasized travelling to China, the family language policy, and parental roles on CHL maintenance, the less likely they sent their children to the Chinese Weekend School. Nevertheless, the emphasis on children's academic work did not have significant differences among the four classifications. However, this statement does not fit for parents with business professional backgrounds.

\subsection{Reasons for the Consistency and Inconsistency Between Attitudes and Effort}

Reference [20] proposed the concepts of voluntary immigrants and involuntary immigrants to explain immigrants' different perspectives on their home language and culture and the dominant language and culture. According to Ogbu's theory, voluntary immigrants were more likely to assimilate to the dominant culture and more willing to learn and increase their proficiency level of the dominant language due to their desire for a brighter future in the new environment [20, 22].

The Chinese-American parents in this study were defined as voluntary immigrants because they voluntarily chose to move to the United States for better economic status and better education for their later generations. In that case, though they keep positive attitudes toward CHL maintenance due to their close connection to China and advanced development of Chinese, they were more willing to support their children's schoolwork instead of assigning home-learning time for Chinese learning. From their perspectives, though their children would benefit from learning Chinese, fluent English and excellent schoolwork will help their children gain access to higher economic status and better education [21, 24].

The reasons of the consistency between grandparents' attitudes and efforts regarding CHL maintenance and the relationship between children's better learning outcome as shown in the YCT test and their grandparents' Chinese instruction at home could be explained as follows: (1) besides holding strong and positive attitudes toward CHL maintenance, they speak Chinese at home because of their incompetence of English speaking; (2) due to limited English proficiency they could only help with their grandchildren's Chinese learning; (3) retired grandparents have more time and effort to place on children's Chinese learning than parents with either university or business occupations.

\subsection{Findings from the Chinese School}

According to the class observation at the Chinese Weekend School, although the Chinese teachers had no previous teaching experience, the teachers were responsible for delivering Chinese language instruction to the children. They were very patient in class to answer questions and correct students' errors. They made every effort to attract students' attention. For example, Hua, as a volunteer Chinese teacher and a full-time mom, who taught 6 to 8 -year-old children used cartoon-film-dictation in class, which motivated students significantly. When doing cartoon-film-dictation, students were asked to write the Chinese characters relating to the content of the cartoon film. The teacher paused the film when students needed to write down the Chinese characters. She told me that before she introduced cartoon-film-dictation to the class children did not like dictation because writing was their weakest skill, and they did not want to practice it. However, cartoon-film made students like the "boring" dictation. What is more, since most of the Chinese characters are pictographic, cartoon-film-dictation put the characters into context, which should increase students' dictation accuracy. However, when asking if she introduced this effective teaching technique to other Chinese teachers, Hua responded with a little bit hesitation.

Excerpt 7

Hua: Introduce the "cartoon dictation" to the other teachers? (Long pause with silent thinking.) I've never thought about this. I mean, there is no teacher training or certain circumstances for this kind of conversation. I have no idea about how and when I could share this with other teachers. Em... I really don't know.

Interviews with parents of the Chinese school group reported that the children liked going to the Chinese Weekend School because it was an extra opportunity for them to be with their best friends. However, the researchers found many teaching disadvantages coexisted with the aforementioned advantages. First, the types of assignments were very traditional and dull. To be frank, they were the least favorite types for the children such as copying text or characters and reading the text aloud three times. What is worse, parents usually did not attach importance to Chinese Weekend School assignments. Without proper supervision at home, completion of assignments was terrible. According to teachers' interviews, they reached a consensus that assignments were meaningless because students seldom touched the Chinese textbooks after school.

Second, though there were some interesting class designs, such as cartoon-film-dictation, there was a lack of classroom activity diversity. In addition, most classroom instruction was humdrum. For example, according to the class observation notes, $\mathrm{Li}$, who was both the Chinese teacher and a graduate student of the local university, only used five simple sentences to instruct students in a one-hour class, such as "read after me," "write down the character," "how do you read this character," "read it louder, I can't hear you," and "look at the blackboard." During the interview, Li was asked how she usually prepare for teaching. Stated:

Excerpt 8

Li: I don't really do any formal preparation. Cause, you know. I am Chinese native-speaker, and I am teaching kids, like aged 6 to 8 . It is very simple. Nothing difficult. I can handle it.

$\mathrm{Li}$ also shared reasons for her teaching in the Chinese school other than her daughter's enrollment. 


\section{Excerpt 9}

Li: For the two hours that my daughter study at the Chinese school, I can also earn some money by teaching Chinese. You know, that is a good thing. It won't take that much extra time to prepare for teaching. Although the salary is $\$ 10 /$ hour, it is not a good salary, but it is better than nothing. Both my husband and I are graduate students, and we both need to do something extra to support the family.

Third, no one followed the classroom language policy, including the teachers. At the very beginning or in the middle of the class, teachers always reminded students of the Chinese only classroom language policy. However, the teachers were unconsciously using English words in their class instructions. In one of the classes, Hua used English 25 times in a one-hour class. The other teacher, Li, used English 18 times. When the researchers reported this to them during the interview, they were astonished. They were not consciously aware of their English use.

Next, the classroom language learning environment could be improved. According to the observation, all the students were called by their English names instead of their Chinese names. When the teachers being asked why they did not call the students by their Chinese names, they demonstrated that it was not necessary to use Chinese names since both they and the students were getting used to being called by English names. Nevertheless, [66] noted the importance of self-identity in HL maintenance. Children will acknowledge their original Chinese identity when being called by Chinese names because the learning context becomes more positive for language learning.

Finally, the Chinese teachers need to develop their classroom management skills and strengthen their teaching methodology. With slim teaching experience, there was weak classroom management alongside the teaching process. Observational notes taken during the observations are described as follows. First, teachers easily got distracted by students' unexpected behavior. It was hard for them to go back to the normal teaching process once being distracted. Second, classroom punishment did not work effectively, students sometimes were confused as to why they were being punished. As Chinese-American children understand both Chinese and American culture, the reason of students' confusion about the punishment had something to do with culture conflicts. For example, Tella, a student of the Weekend Chinese School, was kneeling on the chair when she was writing. The teacher, Hua, asked her to sit on the chair properly. Tella did not pay attention to this and still kneeled. Hua then asked her to stand at the back of the classroom. Tella argued that her teacher at school would allow her to kneel on the chair. She was confused as to why she needed to be punished only because she was kneeling on the chair while writing. In American culture, students kneeling on the chair when writing is not serious enough to be punished, whereas in Chinese culture, students are expected to sit properly on the chair at all times during the class. Because these children have grown up in the United States, their values are closer to American rather than Chinese.
Additionally, CHL schools in large Chinese communities have better teaching outcomes because firstly, they are able to include more versatile curriculum, such as calligraphy, history, geography, folk dance, singing, chess, origami, martial arts, brush painting, public speaking, drama, cooking, and Chinese silk knots [67]. Secondly, some of them establish collaborative relationships with the United States educational system, such as K-12 public and private schools, colleges and universities, and study abroad programs [68]. Finally, some of the Chinese language schools have been granted credit transfer status, which encourages greater student enrollment [36].

\section{Conclusion}

Previous research noted the positive influence of Chinese bilingual education in the United States [66]. In [6], the compliment of Chinese schools refers to those located in cities with large Chinese populations and numerous Chinese communities. Contrary to previous research, this study explores the CHL maintenance in a small Chinese community, which expanded the research area of HL maintenance. According to the SPSS and the interview as well as class observation data analysis of the current study, Chinese schools in small cities are not as effective as the ones located in cities with a larger population of Chinese individuals. Furthermore, even grandparents performed a better job in language teaching than that received in Chinese school.

Additionally, previous research indicates that objective factors facilitating HL maintenance include close connection between community-based Chinese language schools in big metropolitan areas [1], more traditional cultural celebration events $[1,50]$, a number of school districts with large Chinese communities grant credit to students who study at Chinese-language schools [36]. Referring to small cities with limited Chinese population, the HL maintenance situation is not as positive as described in previous research articles.

What is more, the inconsistency between parents' attitudes and their real efforts toward HL learning was not a new finding [35, 69]. However, the reasons for inconsistencies have not been explored adequately. This study explained inconsistencies by combining parents' perspectives of Chinese traditional cultural background with their professional background and their status as voluntary immigrants. To conclude, in order to develop the HL maintenance in small Chinese communities in the United States, parents' positive attitudes towards it is just the title of a book, for their real effort of passing on HL to their children decides the content of the book.

\section{Recommendations}

Because of the unfriendly environment of $\mathrm{HL}$ maintenance in small Chinese communities, Chinese parents should guarantee their children enough exposure to Chinese, for instance, organizing family gatherings, daily exposure of Chinese TV programs, and reading Chinese stories [35] and 
establish an appropriate family language policy, otherwise CHL will gradually experience a language shift rather than maintenance in small Chinese communities in the United States. However, since changing the CHL learning environment in small Chinese communities could not be accomplished within a short period of time, future research could investigate how the local K-12 public schools may facilitate children's HL learning, for example welcoming home-language at school and classroom teachers' positive attitudes toward language and cultural diversity. In addition, since Chinese parents generally retain positive attitudes toward CHL maintenance [3], it is still largely unknown how they put their positive beliefs into home practices [5].

As a limitation of this study, more in-depth qualitative data analysis could have been conducted in order to investigate the difficulties that Chinese parents might have in carrying out effective CHL home practices, besides the impact of their cultural-ecological perspective on HL development. In addition, this research needs to be replicated in various settings to further inform how language maintenance works in these different settings and answer questions concerning how schools in the United States implement policies to facilitate the maintenance of heritage language for the purpose of English learners' academic success, how schools incorporate the heritage language and culture into the curriculum, and what the schools' attitudes and real efforts related to this are. Besides investigating how English learners benefit from their heritage language development, it is important to evaluate the effectiveness of the United States government's investment in foreign language programs.

\section{References}

[1] Li, M. (2005). The role of parents in Chinese heritage-language schools. Bilingual Research Journal 29 (1): 197-207.

[2] Liu, N. (2010). Chinese heritage language schools in the United States. Heritage Briefs Collection, Center for Applied Linguistics.

[3] Lao, C. (2004). Parents' attitudes toward Chinese-English bilingual education and Chinese-language use. Bilingual Research Journal 28 (1): 99-121.

[4] Li, X. (1999). How can language minority parents help their children become bilingual in familial context? A case study of a language minority mother and her daughter. Bilingual Research Journal 23 (2-3): 211-223.

[5] Li, G. (2006). Culturally contested pedagogy: Battles of literacy and schooling between mainstream teachers and Asian immigrant parents. Albany, NY: SUNY Press.

[6] Mu, G. M. and Dooley, K. (2015). Coming into an inheritance: Family support and Chinese heritage language learning. International Journal of Bilingual Education and Bilingualism 18 (4): 501-515.

[7] Xu, L. and Moloney, R. (2014). Identifying Chinese heritage learners' motivations, learning needs and learning goals: A case study of a cohort of heritage learners in an Australian
University. Language Learning in Higher Education 4 (2): 365-393.

[8] Zhang, D. and Slaughter-Defoe, D. T. (2009). Language attitudes and heritage language maintenance among Chinese immigrant families in the USA. Language, Culture and Curriculum 22 (2): 77-93.

[9] Zhang, J. (2009). Chinese heritage language maintenance: A grandparents' perspective (Unpublished master's thesis). McGill University, Montreal.

[10] Echeverria-Estrada., C. and Batalova, J. (2020, January 15). Chinese immigrants in the United States. Migration Policy Institute. Retrieved from https://www.migrationpolicy.org/article/chinese-immigrants-u nited-states

[11] Jia, G. (2008). Heritage language development, maintenance, and attrition among recent Chinese immigrants in New York City. In A. W., He, \& Y. Xiao (Eds.), Chinese as a heritage language: Fostering rooted world citizenry (pp. 189-203). Honolulu, HI: University of Hawai'i, National Foreign Language Resource Center.

[12] He, A. W. (2008). Chinese as a heritage language: An introduction. In A. W. He \& Y. Xiao (Eds). Chinese as a heritage language: Fostering rooted world Citizenry (pp. 1-12). Honolulu: University of Hawai'I Press.

[13] Luo, S. and Wiseman, R. L. (2000). Ethnic language maintenance among Chinese immigrant children in the United States. International Journal of Intercultural Relations 24 (3): 307-324.

[14] Tannenbaum, M. and Howie, P. (2002). The association between language maintenance and family relations: Chinese immigrant children in Australia. Journal of Multilingual and Multicultural Development 23 (5): 408-424.

[15] Yu, S. (2010). How much does parental language behaviour reflect their language beliefs in language maintenance? Journal of Asian Pacific Communication 20 (1): 1-22.

[16] Chiang, P. S. (2010). Home literacy education of Taiwanese Australian families: A sociological analysis (Doctoral dissertation). Queensland University of Technology, Brisbane.

[17] Dai, J. E. and Zhang, L. (2008). What are the CHL learners inheriting? Habitus of the CHL learners. In A. W., He, \& Y. Xiao (Eds.), Chinese as a heritage language: Fostering rooted world citizenry (pp. 37-49). Honolulu: University of Hawai'i Press.

[18] Lawton, B. L. and Logio, K. A. (2009). Teaching the Chinese language to heritage versus non-heritage learners: parents' perceptions of a community weekend school in the United States. Language, Culture and Curriculum 22 (2): 137-155.

[19] Xiao, Y. (2006). Heritage learners in the Chinese language classroom: Home background. Heritage Language Journal 4 (1): 47-56.

[20] Ogbu, J. U. (1978). Minority education and caste: The American system in cross-cultural perspective. New York: Academic Press.

[21] Ogbu, J. U. (1998). Voluntary and involuntary minorities: A cultural-ecological theory of school performance with some implications for Education. Anthropology \& Education Quarterly 29 (2): 155-188. 
[22] Shibutani, T. and Kwan, K. M. (1965). Ethnic stratification: A comparative approach. The MacMillan Co.

[23] Gibson, M. (1988). Accommodation without assimilation: Sikh immigrants in an American high school. Ithaca, NY: Cornell University Press.

[24] Ogbu, J. U. (1995). Cultural problems in minority education: Their interpretations and consequences - Part one: Theoretical background. The Urban Review 27 (3): 189-205.

[25] Curdt-Christiansen, X. L. (2014). Family language policy. In X L. Curdt-Christiansen \& A. Hancock (Eds.), Learning Chinese in diasporic communities: Many pathways to being Chinese (pp. 35-58). Amsterdam, The Netherlands: John Benjamins.

[26] Wiley, T. G. and Valdés, G. (2000). Editors' introduction: Heritage language instruction in the United States: A time for renewal. Bilingual Research Journal 24: i-v.

[27] Shin, S. J. (2010). What about me? I'm not like Chinese but I'm not like American: Heritage-Language learning and identity of mixed-heritage adults. Journal of Language, Identity, and Education 9 (3): 203-219.

[28] Valdés, G. (2001). Learning and not learning English: Latino students in American schools. New York, NY: Teachers College Press.

[29] Van Deusen-Scholl, N. (2003). Toward a definition of heritage language: Sociopolitical and pedagogical considerations. Journal of Language, Identity, and Education 2 (3): 211-230.

[30] Wan, G. (2000). A Chinese girl's storybook experience at home. Language Arts 77 (5): 398-405.

[31] Xu, H. (1999). Young Chinese ESL children's home literacy experiences. Reading Horizons 40 (1): 47-64.

[32] Buckwalter, J. K. and Lo, Y. G. (2002) Emergent biliteracy in Chinese and English. Journal of Second Language Writing 11 (4): 269-293.

[33] Hu, R. and Commeyras, M. (2008). A case study: Emergent biliteracy in English and Chinese of a 5-year-old Chinese child with workless picture books. Reading Psychology 29 (1): 1-30.

[34] Liu, Y. and Vadeboncoeur J. A. (2010). Bilingual intertextuality: The joint construction of biliteracy practices between parent and child. Min, Culture, and Activity 17 (4): 367-381.

[35] Lv, C. and Koda, K. (2011). The impact of home language and literacy support on English-Chinese biliteracy acquisition among Chinese heritage language learners. Heritage Language Journal 8 (2): 199-235.

[36] Chao, D. (1997). Chinese for Chinese Americans: A case study. Journal of the Chinese Language Teachers Association 32 (2): $1-13$.

[37] Wen, X. (2011). Chinese language learning motivation: A comparative study of heritage and non-heritage learners. Heritage Language Journal 8 (3): 41-66.

[38] Fishman, J. A. (1991). Reversing language shift. Clevedon, UK: Multilingual Matters.

[39] Hakuta, K. and d'Andrea, D. (1992). Some properties of bilingual maintenance and loss in Mexican background in high school students. Applied Linguistics 13 (1): 72-99.

[40] Hakuta, K. and Pease-Alvarez, L. (1994). Proficiency, choice, and attitudes in bilingual Mexican-American Children. In G. Extra \& L. Verhoeven (Eds.), The cross-linguistic study of bilingual development (pp. 145-164). New York: North-Holland.

[41] He, A. W. (2010). The heart of heritage: Sociocultural dimensions of heritage language learning. Annual Review of Applied Linguistics 30: 66-82.

[42] Gardner, R. C. and Lambert, W. E. (1972). Attitudes and motivation in second-language learning. Rowley, MA: Newbury House.

[43] Unger, J. (1993). Urban families in the eighties: An analysis of Chinese surveys. In D. Davis \& S. Harrell (Eds.), Chinese families in the post Mao era (pp. 25-49). Berkeley, CA: University of California Press.

[44] Sakamoto, M. (2006). Balancing L1 maintenance and L2 learning. In K. Kondo-Brown (Ed.), Heritage language development: Focus on East-Asian immigrants (pp. 33-56). Amsterdam: John Benjamins.

[45] Shin, S. J. (2005). Developing in two languages: Korean children in America. Clevedon, England: Multilingual Matters.

[46] Siu, S. F. (1993). Taking no chances: A profile of a Chinese-American family's support for school success. Equity and Choice 10 (2): 23-32.

[47] Wu, H. P., Palmer, D. K., and Field, S. L. (2011). Understanding teachers' professional identity and beliefs in the Chinese heritage language school in the USA. Language, Culture and Curriculum 24 (1): 47-60.

[48] Lai, H. M. (2000). Retention of the Chinese heritage. Chinese America: History and Perspectives, 10-27.

[49] Shibata, S. (2000). Opening a Japanese Saturday school in a small town in the United States: Community collaboration to teach Japanese as a heritage language. Bilingual Research Journal 24 (4): 333-342.

[50] Wang, X. (Ed.). (1996). A view from within: A case study of Chinese heritage community language schools. College Park, MD: National Foreign Language Center.

[51] Chao, T. H. (1996). Overview in X. Wang (Ed.), A view from within: A case study of Chinese heritage community language schools in the United States (pp. 7-14). Washington, DC: National Foreign Language Center.

[52] Compton, C. J. (2001). Heritage language communities and schools: Challenges and recommendations. In J. K. Peyton, D. A. Ranard, \& S. McGinnis (Eds.), Heritage languages in America: Preserving a national resource (pp. 145-165). McHenry, IL: Delta Systems.

[53] Jia, Y. and Fan, P. (Eds.) (2002). Zhong Wen (Chinese language). Guangzhou, China: Jinan University Publishing House.

[54] Mu, G. M. (2014a). Heritage language learning for Chinese Australians: The role of Habitus. Journal of Multilingual and Multicultural Development 35 (5): 497-510.

[55] Mu, G. M. (2014b). Learning Chinese as a heritage language in Australia and beyond: The role of capital. Language and Education 28 (5): 477-492.

[56] World Population Review. (2020). Starkville, Mississippi population 2020. Retrieved from https://worldpopulationreview.com/us-cities/starkville-ms-pop ulation/ 
[57] Proximity. (2020, July 4). Mississippi school district demographic characteristics. Retrieved from http://proximityone.com/sd_ms.htm

[58] Fraenkel, J. R., Wallen, N. E., and Hyun, H. H. (2015). How to design and evaluation research in education. $\left(9^{\text {th }}\right.$ ed). New York, NY: McGraw-Hill Education.

[59] Marshall, C. and Rossman, G. B. (2011). Designing qualitative research. Thousand Oaks, CA: SAGE.

[60] Creswell, J. W. (2007). Qualitative inquiry and research design: Choosing among five approaches ( $2^{\text {nd }}$ ed.). Thousand Oaks, CA: Sage.

[61] Kiang, L. (2008). Ethnic self-labeling in young American adults from Chinese backgrounds. Journal of Youth and Adolescence 37 (1): 97-111.

[62] Pao, D. L., Wong, S. D., and Teuben-Rowe, S. (1997). Identity formation for mixed-heritage adults and implications for educators. TESOL Quarterly 31 (3): 662-631.

[63] Chung, Y. Y. (2007). An analysis of Chinese parental attitudes toward their children's heritage language maintenance and development (Doctoral dissertation).
[64] Wajnryb, R. (1992). Classroom observation tasks. Cambridge: Cambridge University Press.

[65] Yu, S. (2014). The immediate effect of parental language choice on that of their children's language in Chinese migrant families. Taiwan Journal of Linguistics 12 (1): 81-108.

[66] Xia, N. (1992). Maintenance of the Chinese language in the United States. Bilingual Review 17 (3): 195-209.

[67] Wang, S. C. (1999). Crossing the bridge: A Chinese case from mother tongue maintenance to foreign language education. In M. Chu (Ed)., Mapping the course of the Chinese language field (pp. 270-312). Chinese Language Teachers' Association Monograph Series, 3.

[68] McGinnis, S. (2008). From mirror to compass: The Chinese heritage language education sector in the United States. In D. M. Brinton, O. Kagan, \& S. Bauckus, (Eds.), Heritage language education: A new field emerging (pp. 229-242). London \& New York: Routledge Taylor \& Francis Group.

[69] Schecter, S. R. and Bayley, R. (2002) Language as cultural practice: Mexican en el Norte. Mahwah, NJ: Lawrence Erlbaurn. 